\title{
Successful Outpatient Management of Pelvic Actinomycosis by Ceftriaxone: A Report of Three Cases
}

\author{
Eda Demir Onal ${ }^{1}$, Akif Altinbas ${ }^{1}$, Ibrahim Koral Onal ${ }^{1}$, Sibel Ascioglu ${ }^{2}$, Meltem Gulsun Akpinar ${ }^{3}$, Cigdem Himmetoglu ${ }^{4}$ and \\ Yesim Cetinkaya Sardan ${ }^{5}$ \\ ${ }^{1}$ Department of Internal Medicine; ${ }^{2}$ Department of Infectious Diseases; ${ }^{3}$ Department of Radiology; ${ }^{4}$ Department of Pathology; ${ }^{5}$ Department of \\ Infectious Diseases; Hacettepe University Medical School; Ankara, Turkey
}

\begin{abstract}
Pelvic actinomycosis is a chronic granulomatous suppurative disease caused by actinomyces israeli. Intravenous penicillin is the preferred antimicrobial but it requires hospitalization up to one month. An outpatient treatment strategy would be cost effective and a good choice for patients. Here we present three cases in which intramuscular ceftriaxone was successfully used in the outpatient settings following surgery and IV penicillin treatment in the hospital.
\end{abstract}

Key-Words: Ceftriaxone, actinomycosis, penicillins, pelvic neoplasms, intrauterine devices.

Actinomycosis is an infection with Actinomyces species, a Gram-positive, anaerobic, non-acid-fast filamentous bacterium. The craniocervical localization arising from oral cavity, is well known. There may be thoracic, abdominal and genital involvement as well. The overall incidence of registered cases of actinomycosis is decreasing. But abdominal and genital actinomycosis are increasing in frequency [1,2].

Pelvic actinomycosis is a chronic granulomatous suppurative disease caused by actinomyces israeli. The infection mimics ovarian tumors during presentation and it is diagnosed only after surgery in most of the cases. Intravenous (IV) penicillin is the preferred antimicrobial ${ }^{3}$ but it requires hospitalization up to one month. An outpatient treatment strategy would be cost effective and a good choice for patients. Here we present three cases in which intramuscular (IM) ceftriaxone was successfully used in the outpatient settings following IV penicillin treatment in the hospital.

\section{Case Reports \\ Case 1}

A 45-year-old woman was admitted to our infectious disease clinic with fever. She had been wearing an intrauterine device (IUD) for fifteen years and it was removed four months ago. On physical examination there was no remarkable finding except for a body temperature of $39^{\circ} \mathrm{C}$. Her erythrocyte sedimentation rate (ESR) was $115 \mathrm{~mm} /$ hour. Chest radiogram and urinalysis revealed no positive finding. An abdominal ultrasonography showed bilateral pelvic masses. On the computerized tomography (CT) no differentiation could be made between ovarian tumor and abscess. She was administered sulbactam/ampicillin $1.5 \mathrm{~g}$ IV every 6 hours and underwent laparotomy after two weeks. In the operation bilateral ovarian abcesses were determined and drained. Then bilateral salpingoophorectomy was performed. Pathologic

Received on 18 May 2009; revised 17 July 2009.

Address for correspondence: Dr. Ibrahim Koral Onal. Tel: 903123061334. E-mail: koralonal@yahoo.com Postal Address: Türkiye Yüksek Ýhtisas Eðitim ve Arabtýrma Hastanesi Gastroenteroloji Servisi Sýhhiye/ Ankara/Turkey PC: 06100.

The Brazilian Journal of Infectious Diseases 2009;13(5):391-393. (C) 2009 by The Brazilian Journal of Infectious Diseases and Contexto Publishing. All rights reserved. examination of the specimens disclosed inflammatory cell infiltration with the predominance of neutrophils and sulfur granules. She was discharged with ceftriaxone IM $2 \mathrm{~g}$ per day. One month later there was no pelvic collection or residual abscess on CT. The patient had no complain with a normal ESR in the laboratory examination. The treatment was stopped. There was no sign of recurrence in the following control visits.

\section{Case 2}

A 32-year-old-woman complained of abdominal pain and fever. She had been using IUD for about 6 years and she had it removed one month before. In the blood count there was a mild leucocytosis with 12,000/uL and her ESR was $39 \mathrm{~mm} / \mathrm{h}$. On the physical examination there was a fever of $39^{\circ} \mathrm{C}$ and a mild diffuse abdominal sensitivity. An abdominal ultrasonography showed unilateral tuboovarian abcess and unilateral salpingoophorectomy was performed. In the pathological examination of the specimen sulfur granules were observed. We started daily intravenous crystallized penicillin G 24 million IU over a 24-hour period of time. We discharged the patient after one week of IV penicilin therapy with IM ceftriaxone $2 \mathrm{~g}$ per day. She had no complain one month later with normal leucocyte count, ESR and CT findings. The treatment was stopped. No sign of recurrence was observed in the control visits.

\section{Case 3}

A 55-year-old woman was referred to gynecology clinic because of fever and pelvic pain. Her past medical history included crohn's disease and she was under treatment with steroids and azathiopurine. On physical examination she had a fever of $40^{\circ} \mathrm{C}$. Her pelvic examination was painful. She had a leucocytosis with 13,000/uL and mildly elevated serum CA125 level. In her pap smear sulfur granules were seen and pelvic CT scan revealed pseudocystic changes on the left adnexial area (Figure 1). Total abdominal histerectomy and left salpingoophorectomy was made. Multiple small abcesses were observed in the myometrium and endometrium. In the pathologic examination sulfur granules were seen (Figure 2). After surgery we started daily intravenous crystallized penicillin G 24 million IU over a 24-hour period of time. We discharged the patient after one month of IV penicilin therapy 
Figure 1. Pelvic actinomycosis: Pseudocystic lesion on the left adnexial area in computerized tomography (indicated by arrow).

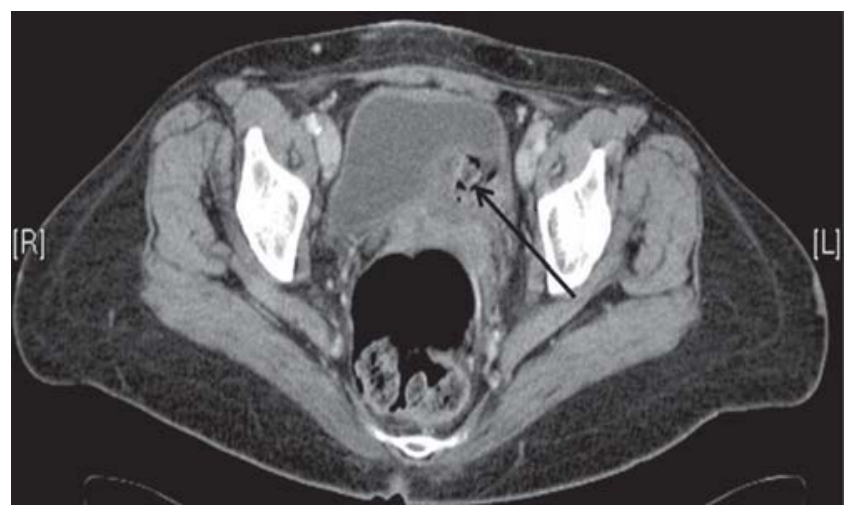

Figure 2. Actinomycosis: Inflammatory cells with the predominance of neutrophils and sulfur granules (indicated by arrows).

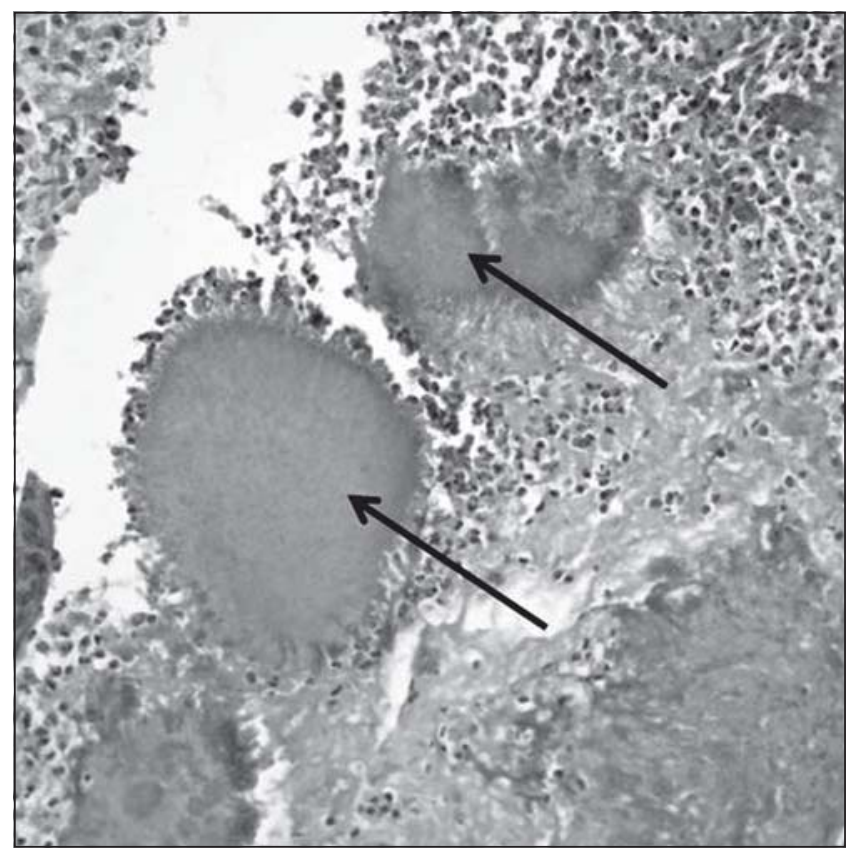

with IM ceftriaxone 2 g per day. In the control visit after 6 weeks she had no complain with normal leucocyte count and CT findings (Figure 3). Ceftriaxone was stopped and she was followed up with no signs of recurrence.

\section{Discussion}

The actinomyces is an anaerobic slow growing Grampositive bacillus that can be found in the normal flora of the body and is merely associated with infections in the general population. A characteristic granulomatous inflammatory response, with pus production and abcess formation, followed by necrosis and extensive reactive fibrosis is formed by the microorganism. Hematogenous spread is rare and lymphatic dissemination does not occur [1].
Figure 3. Pelvic actinomycosis: In computerized tomography there is no residual abscess or collection on the left adnexial area after treatment.

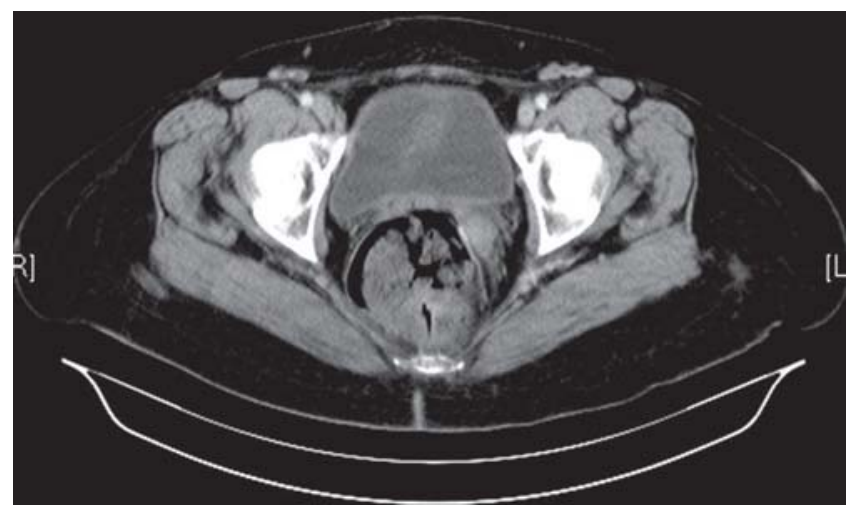

Destruction of the mucosal barriers, e.g. by trauma, endoscopic manipulations, operations or chronic inflammatory diseases are predisposing factors for penetration of pathogens into deep tissues [4,5].

Though frequently cited [6], the association between IUCD use and development of pelvic actinomycosis is a controversial one. It is accepted that the risk of pelvic actinomycosis resulting from IUCD use is very low. However, it has been suggested that the risk of pelvic actinomycosis may be higher with prolonged use of an IUCD [7]. Our two patients were using IUD for more than 6 years.

It is difficult to distinguish the colonisation of the female genital tract with A.israeli and the real pelvic actinomyces infection. In the colonisation there is no symptom, but in the pelvic actinomycosis the symptoms of endocervicitis, endometritis, salpingitis or tubo-ovarian abscess can be seen. In our three cases there were pelvic abcesses and related symptoms.

Preoperative clinical diagnoses of pelvic actinomycosis is really difficult. The clinical and laboratory findings are nonspesific. Presenting signs and symptoms are lower abdominal mass and pain, vaginal discharge or hematuria. Occasionally fever and elevated white cell count or ESR are found [8]. However all of our 3 patients have leucocytosis and elevated erythrocyte sedimentation rates, fever and abdominal pain.

Histopathologic examination of the infected tissue should include a search for characteristic appearances of sulfur granules (Figure 2). The granules measure 40-400 ìm and stain Gram-positive with a mycelium-like structure. We found sulfur granules in all our patients during histopathological examination. Culturing of actinomyces species requires freshly obtained material and transporting should be done in specific, anaerobic containers immediately [9]. Despite suitable conditions culture is negative in $76 \%$ of actinomyces cases. We could not grow the microorganism in the two of our three cases in which we sent tissue for culture.

Actinomyces species are susceptible to most antibiotics [9]. Generally the recommendation is to initiate treatment with 
intravenous penicillin G (20 million IU/day) for four weeks followed by oral penicillin V (2-4 g/day) for 2-12 months [10]. In cases of penicillin intolerance, clindamycin or tetracycline can be used with good results [8]. Regarding the long hospitalisation time required for IV penicilin therapy there is a need for new antimicrobials in the treatment of actinomycosis which may facilitate outpatient management. Considering the data in the literature on successful therapy of actinomycosis with ceftriaxone this drug may be a good candidate for outpatient approach because it can be administered intramuscularly once in a day. Among the few case reports are thoracic [10,11], oesophageal [12], cerebral [13] and urinary bladder [14] actinomycotic infections in which ceftriaxone was used alone, following surgery or IV penicillin therapy. In the first of our cases we used ceftriaxone following only two weeks of IV sulbactam/ampicillin. In the second initial treatment period with IV penicillin was one week. In the third case the hospitalisation period was prolonged to one month because of her inflammatory bowel disease requiring inpatient management. During this time IV penicilin was administered. In all three cases the duration of treatment was less than two months with no sign of recurrence in the follow-up. To the best of our knowledge this is the first case series in the literature IM ceftriaxone was successfully used in the management of pelvic actinomycosis as an adjunctive therapy to surgery and short term IV penicilin.

It should be reminded that a surgical procedure is almost always necessary in actinomycosis. The surgery is needed when the pelvic abcess is widening despite the IV antibiothreapy, in the presence of compression on the urinary tract or bowel or when there is a high suspicion of pelvic malignencies. It is really difficult to diagnose pelvic actinomycosis before surgical approach. In the literature the majority of the diagnosis were reached after the explorative laparotomy usually with the suspicion of malignancies.

In conclusion daily IM ceftriaxone therapy can facilitate outpatient management of pelvic actinomycosis. Whether there is a need for an initial IV penicilin treatment and the duration of therapy with ceftriaxone would become more clear in the future with the accumulation of evidence on the subject.

\section{References}

1. Yegüez J.F., Martinez S.A., Sands L.R., Hellinger M.D. Pelvic actinomycosis presenting as malignant large bowel obstruction:a case report and a review of the literature. Am Surg 2000;66:8590 .

2. Kabwa L., Mattei J., Ramadane A. Actinomycose abdominale. J Chir(Paris) 1996;7: 348-52.

3. Taga S. Diagnosis and therapy of pelvic actinomycosis. J Obstet Gynaecol Res 2007;33: 882-5.

4. Jung C., Wagner J., Heine B. et al. Abdominelle Aktinomykose nach Magen-operation bei einer Patientin mit langjahriger rheumatoider arthritis unter methotrexat-therapie. Dtsch Med Wschr 1999;124:1066-70.

5. Harsch I.A., Benninger J., Niedobitek G. et al. Abdominal actinomycosis: complication of endoscopic stenting in chronic pancreatitis? Endoscopy 2001;33:1065-9.

6. Dharmadhikari D., Dharmadhikari R., Macdonald J. et al. Intrauterine contraceptive device-related actinomycosis infection presenting as incarcerated inguinal hernia. J Obstet Gynaecol Res 2007;33:595-7.

7. Evans D.T. Actinomyces israelii in the female genital tract: a review. Genitourin Med 1993;69:54-9.

8. Wagenlehner F.M., Mohren B., Naber K.G., Männl H.F. Abdominal actinomycosis. Clin Microbiol Infect 2003;9: 881-5.

9. Koneman E.W., Allen S.D., Janda W.M., Schreckenberger, Winn W.C. Jr eds. Color Atlas and Textbook of Diagnostic Microbiology, 5th edn. Philadelphia: Lippincott-Raven, 1997.

10. Ferrari T.C., Couto C.A., Murta-Oliveria C et al. Actinomycosis of the colon: a rare form of presentation. Scand J Gastroenterol 2000;1:108-9.

11. Skoutelis A., Petrochiolis J., Bassaris H. Successful treatment of thoracic actinomycosis with ceftriaxone. Clin Infect Dis 1994;19:161-2.

12. Abdalla J., Myers J., Moorman J. Actinomycotic infection of the oesophagus. Journal of infection 2005;51:39-43.

13. Olah E., Berger C., Boltshauser E., Nadal D. Cerebral actinomycosis before adolescence. Neuropediatrics 2004;35:239-41.

14. Aguirrebengoa K., Aruza A., Bececiartura E., Montejo M. Primary actinomycosis of the urinary bladder. Scand J Infect Dis 2000;32:330-1. 